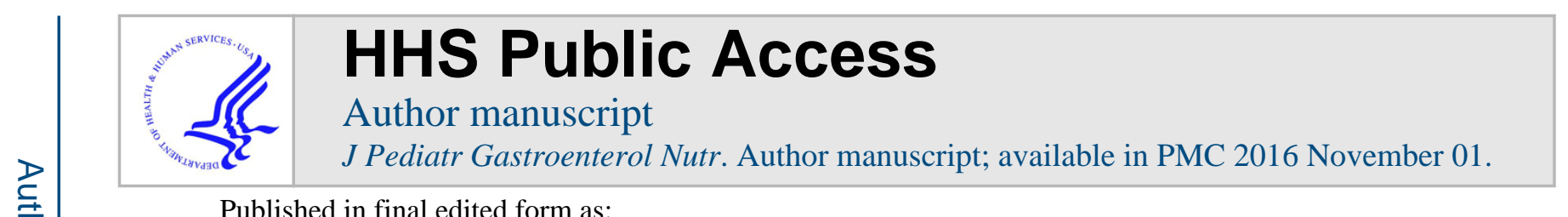

Published in final edited form as:

J Pediatr Gastroenterol Nutr. 2015 November ; 61(5): e26. doi:10.1097/MPG.0000000000000959.

\title{
Response to DEFI-ALPHA Cohort
}

\author{
Jeffrey H Teckman 1 \\ ${ }^{1}$ Pediatrics and Biochemistry, Saint Louis University, Cardinal Glennon Children's Medical \\ Center, Saint Louis, MO, United States. teckmanj@slu.edu
}

\section{Keywords}

cirrhosis; liver transplant; liver enzymes; metabolic liver disease; jaundice

Dear Drs. Ruiz, Joly and Lachaux.

It is exciting to hear about the DEFI-ALPHA cohort. We agree that a possible explanation for the somewhat different rate of severe disease might be a different set of modifier genes in the North American population. However, there are other significant differences in the two cohorts, some of which you mention, which make it very difficult to compare. First, the referral patterns in the US of ZZ patients with liver disease to the ChiLDREN study centers are likely very different than the referrals to DEFI-ALPHA. This might be due to the variability of the US insurance system, the functions of the medical system within different states in the US and the function of private versus academically affiliated US medical centers. The ChiLDREN cohort is not a representative, population based sample. Another important factor is the defined DEFI-ALPHA inclusion system which is based on serum level and not phenotype. The serum level is not directly related to liver disease, as the liver injury is related to accumulation of the $\mathrm{Z}$ protein within hepatocytes, not the lack of circulating protein. It is somewhat puzzling to include PISS patients in a liver disease cohort since most authorities do not regard PISS as susceptible to liver disease. While the S mutant protein is retained within the liver, it does not form the toxic, intrahepatic polymers unless present with the $\mathrm{Z}$ mutant protein, as is the case in the PISZ individual. Furthermore, inclusion in DEFI-ALPHA of MZ individuals is even more confounding since the incidence of liver disease is MZ heterozygotes (usually called "carriers") appears to be several logs lower than for PIZZ. Many authorities regard MZ as a genetic modifier of other forms of liver injury with minimal risk of liver disease in otherwise well PIMZ individuals. It would be more accurate to report the DEFI-ALPHA cohort rate of severe disease in PISZ and PIZZ individuals, the only well recognized groups with any significant risk of liver disease, and to compare that to the ChiLDREN cohort.

Jeffrey Teckman, MD

Correspondence: Jeffrey Teckman, MD. Professor of Pediatrics and Biochemistry and Molecular Biology, Saint Louis University School of Medicine, 1465 S. Grand Blvd., St. Louis, Missouri 63104 teckmanj@slu.edu FAX: 314-268-2775 Telephone: 314-577-5647.

Clinical Trial Registry: ClinicalTrials.gov: Evaluating the Genetic Causes and Progression of Cholestatic Liver Diseases (LOGIC) NCT00571272. 
Professor of Pediatrics and Biochemistry

Saint Louis University School of Medicine 